\title{
Distinguishing Maps
}

\author{
Thomas W. Tucker \\ Department of Mathematics \\ Colgate University \\ Hamilton, NY, U.S.A.
}

Submitted: Aug 17, 2009; Accepted: Feb20, 2011; Published: Feb 28, 2011

Mathematics Subject Classification: 05E18, 05C10

In memory of Michael O. Albertson

\begin{abstract}
The distinguishing number of a group $A$ acting faithfully on a set $X$, denoted $D(A, X)$, is the least number of colors needed to color the elements of $X$ so that no nonidentity element of $A$ preserves the coloring. Given a map $M$ (an embedding of a graph in a closed surface) with vertex set $V$ and without loops or multiples edges, let $D(M)=D(\operatorname{Aut}(M), V)$, where Aut(M) is the automorphism group of $M$; if $M$ is orientable, define $D^{+}(M)$ similarly, using only orientation-preserving automorphisms. It is immediate that $D(M) \leq 4$ and $D^{+}(M) \leq 3$. We use Russell and Sundaram's Motion Lemma to show that there are only finitely many maps $M$ with $D(M)>2$. We show that if a group $A$ of automorphisms of a graph $G$ fixes no edges, then $D(A, V)=2$, with five exceptions. That result is used to find the four maps with $D^{+}(M)=3$. We also consider the distinguishing chromatic number $\chi_{D}(M)$, where adjacent vertices get different colors. We show $\chi_{D}(M) \leq \chi(M)+3$ with equality in only finitely many cases, where $\chi(M)$ is the chromatic number of the graph underlying $M$. We also show that $\chi_{D}(M) \leq 6$ for planar maps, answering a question of Collins and Trenk. Finally, we discuss the implications for general group actions and give numerous problems for further study.
\end{abstract}

\section{Introduction}

A group $A$ acting faithfully on a set $X$ has distinguishing number $k$, written $D(A, X)=k$, if there is a coloring of the elements of $X$ with $k$ colors such that no nonidentity element of $A$ is color-preserving, and no such coloring exists with fewer than $k$ colors. We also say that an action of $A$ on $X$ is $k$-distinguishable if $D(A, X) \leq k$. The concept was introduced by Albertson and Collins [2] in the context of the automorphism group of a graph acting on the vertex set and extended to general group actions by Tymoczko [25] (see also $[4,5,27]$ ). On the other hand, similar ideas are found earlier in permutation groups; indeed, Bailey and Cameron [3] cite many situations where graph theorists have 
rediscovered and renamed concepts from permutation groups. The graph theoretic origin of distinguishing number [2] is the Necklace Problem: to destroy any symmetry of a necklace of $n$ beads, one needs beads of three different colors for $n=3,4,5$, but only two colors for $n>5$. That is, $D\left(\operatorname{Aut}\left(C_{n}\right), V\left(C_{n}\right)\right)=2$ for $n>5$. The Necklace Problem actually plays a role in some of our proofs.

The generic case for group actions is 2-distinguishability in a variety of contexts; that is, given a group $A$ acting faithfully on a set $X$, one should expect to find a set $Y$ such that the only element of $A$ leaving $Y$ invariant is the identity. For example, this follows immediately in all cases when $A$ is abelian: since point stabilizers are conjugate in $A$, just choose for $Y$ one point in each orbit of $A$. It is true, but much deeper, in all cases when $A$ has odd order, by Gluck's Theorem [10]. Other examples where $D(A, X)=2$ in all but finitely many cases include repeated Cartesian products of a graph $[1,13]$, primitive permutation groups [11, 21], automorphism groups of finite vector spaces or groups [7], transitive actions where the order of $A$ is polynomial in the size of $X$ [7].

A map is an embedding of a graph in a closed surface; throughout, we assume that maps have no multiple edges or loops. This paper studies the distinguishing numbers $D(M)=D(\operatorname{Aut}(M), V)$ or $D^{+}(M)=D\left(\operatorname{Aut}^{+}(M), V\right)$, where $M$ is a map with vertex set $V$ and automorphism group $\operatorname{Aut}(M)$ and, if $M$ is orientable, orientation-preserving automorphism group $\mathrm{Aut}^{+}(M)$. The automorphism group of a map is much more restricted than the automorphism group of the underlying graph, since vertex stabilizers are cyclic or dihedral and edge stabilizers have order at most 4. An immediate consequence is that $D(M) \leq 4$ and $D^{+}(M) \leq 3$ for all maps $M$. The theme of this paper is that, just as in other contexts, the generic situation is $D(M)=2$. It should be noted that this paper, in preprint form, precedes the only other papers on distinguishing maps $[9,15,16]$.

Collins and Trenk [6] have introduced the related idea of distinguishing chromatic number $\chi_{D}(G)$ of graph $G$, where now the distinguishing coloring must also be proper, namely adjacent vertices get different colors. For graphs, $\chi_{D}(G)$ can be arbitrarily larger than the chromatic number $\chi(G)$. We show for maps, just as the generic case for distinguishing number is $D(M) \leq 2$, the generic case for distinguishing chromatic number is $\chi_{D}(M) \leq \chi(M)+2$.

We summarize the major results of this paper:

Theorem 1.1 If $M$ has a vertex of valence 1 or 2 , then $D(M)=2$ unless the underlying graph for $M$ is $C_{n}, K_{1, n}$, or $K_{2, n}$ for $n=3,4,5$.

Theorem 1.2 For all but finitely many maps, $D(M)=2$.

Theorem 1.3 If $A$ is any group of automorphisms of a graph $G$ such that the only element of $A$ fixing adjacent vertices is the identity, then $D(A, V(G))=2$ unless $G$ is $K_{4}, K_{5}, K_{7}$ or the octahedral graphs $\mathrm{O}_{6}, O_{8}$. In particular, the only Frobenius group actions with $D(A, X)>2$ are for $|X|=4,5,7$.

Theorem 1.4 There are only four maps $M$ with $D^{+}(M)>2$ : the tetrahedron, the octahedron, the triangulation of the torus by $K_{7}$, and the quadrangulation of the torus by $K_{5}$. 
Theorem 1.5 For all maps $\chi_{D}(M) \leq \chi(D)+3$, with equality in only finitely many cases.

Theorem 1.6 For all planar maps $\chi_{D}(M) \leq 6$, with equality in only finitely many cases.

We note that a sequel to this paper classifies the graphs underlying the finitely many maps $M$ with $D(M)>2$.

This paper is organized as follows. In Section 2, we summarize for maps the structure of stabilizers for vertices, edges, and "angles" at a vertex. These are the main tools for the rest of the paper. We also discuss the Russell-Sundaram Motion Lemma and prove Theorem 1.1. In Section 3, we use the Motion Lemma to prove Theorem 1.2. In Section 4, we prove Theorem 1.3, which we then use to prove Theorem 1.4. In Section 5, we consider the distinguishing chromatic number for maps, proving Theorems 1.5 and 1.6. The latter answers a question of Collins and Trenk [6]. In Section 6, we consider questions about the distinguishability of graphs, suggested by our work for maps. We also give various problems for further study.

I wish to thank Karen Collins, Marston Conder, Seiya Negami, Alen Orbanič, Tomo Pisanski, Jozef Širáň, Ann Trenk, Mark Watkins, and Steve Wilson for helpful comments. I also wish to thank a referee whose lengthy, careful, and thoughtful review led, I hope, to a much more readable paper.

But this paper really owes its existence to Mike Albertson, who, in his first week at Colgate in 2004 as the Neil Grabois Visiting Professor, came into my office and said: "Let's talk about what math we are doing. I get to go first." He proceeded to tell me about distinguishability. He knew it was a great idea. My immediate response was "Have you tried it on maps?" His untimely death in March 2009 robbed us of any more of his ideas. This paper is dedicated to Mike Albertson.

\section{Map automorphisms and stabilizers}

A map $M$ is an embedding of a graph $G$, called the underlying graph, in a closed surface $S$, called the underlying surface, such that each component, or face, of $S-G$ is homeomorphic to an open disc (that is, the embedding is cellular). In this paper, all maps are connected and finite, with no multiple edges or loops. A map is orientable or not depending on whether the underlying surface is orientable or not. We denote the vertex set of $M$ by $V(M)$. There are a variety of ways of looking at maps as combinatorial structures: rotation systems or band decompositions [12], permutation groups acting on directed edges (monodromy or dart groups)[17], triples of vertex-edge-face incidences (flags) $[24,23]$. Since we are only interested in properties of automorphisms, we will keep our viewpoint intuitive, rather than technical. For our purposes, it is best to think of a map as a dissection of a surface into vertices, edges, and faces.

An automorphism of a map is a homeomorphism of the surface taking vertices to vertices, edges to edges, and faces to faces. We consider two automorphisms to be the same if they define the same bijections of the vertex set, the edge set, and the face set. Since these sets are finite, there are only finitely many automorphisms of a map. The 
collection of all automorphisms of $M$ is a finite group, denoted $\operatorname{Aut}(M)$. If $M$ is orientable, the automorphisms that are orientation-preserving form an index two subgroup denoted $\operatorname{Aut}^{+}(M)$.

Suppose that $u v$ is an edge of map $M$ and the faces on either side of $u v$ are $f$ and $f^{\prime}$. Then if an automorphism fixes $u, v$ and $f$, it must also fix $f^{\prime}$, as well as all vertices and edges incident to $f$, and hence all faces incident to these edges etc. By connectivity, we must have that the automorphism fixes all faces, vertices and edges. Thus the only nonidentity automorphism of $M$ fixing $u$ and $v$ must interchange $f$ and $f^{\prime}$. If $M$ is orientable, this automorphism must be orientation-reversing and can be viewed as a reflection across edge $u v$.

Suppose instead that $v$ is a vertex of a map $M$ of valence $d$. Then any automophism fixing $v$ must take a small disk neighborhood of $v$ to itself. If we view the $d$ edges incident to $v$ as spokes in a wheel, then the automorphism must act on the spokes like an element of the dihedral group $D_{d}$ acting in the usual way on $d$ points on a circle.

To summarize:

- There is at most one nonidentity automorphism fixing adjacent vertices and if the map is orientable, the automorphism is orientation-reversing.

- If vertex $v$ has valence $d$, then there is a cyclic order for the neighbors of $v$ such that any automorphism fixing $v$ acts on the neighbors as an element of the dihedral group $D_{d}$.

We want the action of $A u t(M)$ on the vertex set $V(M)$ to be faithful. This is one reason we require our maps not to have multiple edges or loops. Even with this restriction, consider the map $M$ of a cycle $C_{n}$ lying along the equator of the sphere. Then the reflection interchanging the northern and southern hemispheres is an automorphism of $M$ leaving the equator fixed, so the action of $A u t(M)$ on $V(M)$ is not faithful. We claim this is the only map where the action is not faithful. Indeed, if the map $M$ has any vertex $v$ of valence $d>2$, then any automorphism fixing all vertices would fix all edges incident to $v$ and hence all faces incident to $v$, making the automorphism the identity. The only graphs with all vertices of valence 1 or 2 are paths and cycles. Both have maps only in the sphere. Since there is only one face in the case of a path, the action of $A u t(M)$ is faithful, leaving only the cycle on the equator as a map whose automorphism group does not act faithfully. Since our definition of distinguishing number requires a faithful action, we will deal with the equatorial map separately.

Our graph theoretic notation and terminology are minimal. The $n$-cycle is denoted $C_{n}$. The complete graph on $n$ vertices is denoted $K_{n}$ and the complete bipartite graph on $m$ and $n$ vertices is denoted $K_{m, n}$. For even $n$, we denote by $O_{n}$ the octahedral graph obtained from $K_{n}$ by removing $n / 2$ disjoint edges. The valence of a vertex in a graph or map is the number of edges incident to that vertex. A branch vertex is one of valence greater than 2. The size of a set $Y$ is denoted $|Y|$.

Suppose that $A$ acts on the set $X$ and $Y \subset X$. The (setwise) stabilizer of $Y$ denoted $\operatorname{Stab}(Y)$, is the subgroup of all $a \in A$ leaving $Y$ invariant. That is,

$$
\operatorname{Stab}(Y)=\{a \in A \mid a(y) \in Y \text { for all } y \in Y\} .
$$


The pointwise stabilizer of $Y$, denoted, $\operatorname{Fix}(Y)$, is the subgroup of all $a \in A$ fixing all elements of $Y$. That is,

$$
\operatorname{Fix}(Y)=\{a \in A \mid a(y)=y \text { for all } \in Y\}
$$

In contexts where there may be more than one group action, we write $\operatorname{Stab}_{A}(Y)$ and $\operatorname{Fix}_{A}(Y)$. Note that if $|Y|=1$, then $\operatorname{Fix}(Y)=\operatorname{Stab}(Y)$, and if $|Y|=2$, then $\operatorname{Fix}(Y)$ has index at most 2 in $\operatorname{Stab}(Y)$. We say that $\operatorname{Stab}(Y)$ or $\operatorname{Fix}(Y)$ is trivial if it contains only the identity.

Remark: Note that $D(A, X)=1$ if and only if $A$ is the trivial group. Also, $D(A, X)=$ 2 if and only if $A$ is nontrivial but $\operatorname{Stab}(Y)$ is trivial for some nonempty subset $Y$ of $X$ : simply color $Y$ white and all other elements of $X$ black. Finally, if $\operatorname{Fix}(Y)$ is trivial and $Y$ has $k$ elements, then $D(A, X) \leq k+1$ : just color each element of $Y$ with the first $k$ different colors and color the remaining vertices with the last color.

In terms of this notation, our earlier remarks on automorphisms fixing a vertex or edge can be stated as follows for the action of $\operatorname{Aut}(M)$ on $V(M)$ :

Proposition 2.1 If $u v$ is an edge of the map $M$, then $\operatorname{Fix}(u, v)$ has at most one nonidentity element, which is orientation-reversing if $M$ is orientable.

Proposition 2.2 If $v$ is a vertex of valence $d$ in the map $M$, then its neighbors have cyclic order such that $\operatorname{Stab}(v)$ acts as a subgroup of the dihedral group $D_{d}$.

Given a map $M$, define an angle at $v$ to be a triple of vertices $u v w$ where $u v$ and $v w$ are edges. If $u$ and $w$ correspond to antipodal points in the rotation at $v$, we call the angle straight; otherwise, we call the angle bent. If there is also an edge $u w$ we call the angle $u v w$ closed; otherwise it is open. If $u v$ and $v w$ are consecutive edges in a face boundary incident to $v$, then we call the angle uvw a corner of the embedding. From the dihedral action of $\operatorname{Stab}(v)$ on the neighbors of $v$ we have:

Proposition 2.3 Suppose that uvw is a bent angle. Then $\operatorname{Fix}(u, v, w)$ is trivial. Moreover, there is at most one automorphism, called an angle reflection, fixing $v$ and interchanging $u$ and $w$ and it is an involution; if the map is orientable, such an automorphism is orientation-reversing. In particular, if uvw is an open bent angle, then $\operatorname{Stab}(u, v, w)$ is trivial for $\mathrm{Aut}^{+}(M)$.

We can use the structure of edge and angle stabilizers immediately to get bounds on $D(M)$ and $D^{+}(M)$. If map $M$ has a vertex $v$ of valence greater than 2 , it has a bent angle $u v w$. Thus by Proposition 2.3, $\operatorname{Fix}(u, v, w)$ is trivial so $D(M) \leq 4$. If $M$ is instead a path or cycle, then clearly $D(M) \leq 3$. Also, by Proposition 2.1 , for any edge $u v$, we have $\operatorname{Fix}(u, v)$ is trivial in $\operatorname{Aut}^{+}(M)$, so $D^{+}(M) \leq 3$. Summarizing,

Corollary 2.1 $D(M) \leq 4$ for all maps $M$ and $D^{+}(M) \leq 3$ for all orientable maps $M$. 
It is tempting to try to construct maps with $D(M)>2$ by subdividing edges with extra vertices or by adding pendant vertices in a way that leaves $\operatorname{Aut}(M)$ unchanged. For example, one might try to get around the restriction on multiple edges or loops by subdividing edges. The following theorem shows that such vertices guarantee that $D(M)=2$, except for a few small maps related to the Necklace Problem. Note that to allow discussion of the equatorial map, we must extend the definition of distinguishing number to non-fathful actions: instead of requiring the only color-preserving element to be the identity, we require it to fix all elements of $X$.

Theorem 2.1 If $M$ has at least one vertex of valence 1 or 2 and $D(M)>2$, then the graph underlying $M$ is $C_{n}, K_{1, n}$ or $K_{2, n}$, for $n=3,4,5$.

Proof. Throughout the proof, we let $M$ be a map with $D(M)>2$, so $\operatorname{Stab}(Y)$ is nontrivial for any $Y \subset V(M)$. If $M$ has no branch vertex, making the underlying graph $G$ a path or cycle, we get $G=C_{n}$ for $n=3,4,5$, by the Necklace Problem.

Therefore we assume $M$ has a branch vertex. Suppose that $M$ has a vertex of valence 2. Then it has one, $u$, adjacent to a branch vertex $v$. Since $u$ and $v$ have different valences and $\operatorname{Stab}(u, v)$ is not trivial, there must be a reflection $f$ fixing $u$ and $v$. Since $v$ is a branch vertex, there is a vertex $w$ such that $u v w$ is a bent angle. If $w$ has valence 1 , then $\operatorname{Stab}(u, v, w)$ is trivial, since $u, v, w$ have different valences. If $w$ is a branch vertex, a nontrivial element of $\operatorname{Stab}(u, v, w)$ must interchange $v$ and $w$, since $u$ is not a branch vertex. This forces an edge between $u$ and $w$. For the same reason, there must be an edge from $u$ to $f(w)$, but this contradicts $u$ having valence 2 (note that $f(w) \neq w$ since the angle $u v w$ is bent).

Thus $w$ must have valence 2 . Let $x$ be its other neighbor. Then $x \neq v$ since otherwise $M$ would have multiple edges, and $x \neq u$, since otherwise $w$ and $f(w)$ are both adjacent to $u$. Since $u$ and $w$ have valence 2 and $v$ does not, a nontrivial element of $\operatorname{Stab}(u, v, w, x)$ either interchanges $u$ and $w$, or interchanges $v$ and $x$ or performs a 3-fold rotation of $u, w, x$. In all cases, this forces an edge between $u$ and $x$, so the other neighbor of $u$ is the same as the other neighbor of $w$.

Since $u v w$ was an arbitrary bent angle at $v$ with $u$ having valence 2 , we conclude that all neighbors of $v$ have valence 2 and that they all have the same other neighbor $x$. Repeating the same argument with $x$ instead of $v$, we conclude that the underlying graph $G=K_{2, n}$, and by the Necklace Problem, we must have $n=3,4,5$.

Suppose instead that $M$ has no vertex of valence 2 , but does have a vertex of valence 1. If $M$ has more than one branch vertex, there must be a bent angle $u v w$ where $u$ has valence 1 and $v$ and $w$ are branch vertices. Then $\operatorname{Stab}(u, v, w)$ is trivial, so $D(M) \leq 2$. If $M$ has only one branch vertex, then the underlying graph $G=K_{1, n}$, since all other vertices have valence 1 . By the Necklace Problem, we must have $n=3,4,5$.

We could assume from this point on that there are no vertices of valence 1 or 2 in any of our maps, but we do not because we are also interested in distinguishing graphs and chromatically distinguishing maps, where vertices of valence 2 can be important. 


\section{$3 \quad$ Only finitely many maps have $D(M)>2$}

Before we begin our analysis of maps with $D(M)>2$, we use the Russell and Sundaram Motion Lemma [19] to show that our problem is basically a finite one. Let $A$ act faithfully on the set $X$. Define the motion of an element $a$ of $A$ to be $m(a)=|\{x \mid a(x) \neq x\}|$; define the motion of $A$ on $X$, denoted $m(A)$, to be the minimum of $\{m(a) \mid a \neq 1\}$. The motion of a permutation group is also called the minimal degree (see [8]). Then we have:

Lemma 3.1 (The Motion Lemma) Given A acting faithfully on $X$, if $m(A)>2 \log _{2}(|A|)$, then $D(A, X)=2$

Proof. We sketch the proof since it is so short and elegant. Color $X$ randomly black and white. Suppose that $a \in A$, as a permutation of $X$, has a cycle of length $c$. The probability that all $x$ in that cycle have the same color is $(1 / 2)^{c-1}$. Therefore, the probability that $a$ preserves the coloring is $(1 / 2)^{k}$, where $k$ is the sum of the cycle lengths minus the number of cycles. It is easy to see that $m(a) \leq 2 k$. Thus the expected number of nonidentity elements of $A$ preserving the coloring is at most $(|A|-1)(1 / 2)^{m(A) / 2}$. When $m(A)>2 \log _{2}(|A|)$, the expected number is less than one, guaranteeing at least one coloring that is not preserved by any $a \neq 1$.

The following Lemma gives lower bounds on motion for automorphism groups of maps:

Lemma 3.2 Suppose that $M$ is a map with $n$ vertices, all of valence at least 3 . Let $A=\operatorname{Aut}(M)$, acting on $V(M)$.

a) If all vertices have the same valence, then $m(A) \geq n / 6$.

b) If the maximum valence is $d$, then $m(A) \geq\left(2 / d^{2}\right) n$.

Proof. Suppose all vertices have valence $d$. We count the number of vertices moved by a given nonidentity automorphism. Let uvw be any bent angle. Then at least one vertex in the angle must be moved. If $d$ is odd, there are $d(d-1) n / 2$ such angles. If $d$ is even, there are $(d(d-1) / 2-d / 2) n$ such angles. Every vertex $v$ is in at most $d(d-1) / 2$ angles as the middle vertex and at most $d(d-1)$ angles as an end vertex. Thus the motion of a single vertex $v$ will be counted at most $d(d-1) / 2+d(d-1)$ times. The total number of vertices moved, if $d$ is odd, is then at least:

$$
\frac{d(d-1) / 2}{d(d-1) / 2+d(d-1)} n=\frac{1}{1+2} n=n / 3
$$

and if $d$ is even, at least:

$$
\frac{d(d-1) / 2-d / 2}{d(d-1) / 2+d(d-1)} n=\frac{d-2}{d-1} n / 3>n / 6 .
$$

Suppose instead that the maximum valence is $d$. There are at least $3 n$ bent angles (since every vertex has valence at least 3 ) and the motion of a single vertex is counted at most $d^{2} / 2+d^{2}$ times, giving $m(A)>\left(2 / d^{2}\right) n$. 
The condition on the valences all being the same or bounded is crucial. The double pyramid in the sphere with $C_{n}$ along the equator has an automorphism which moves only the north and south poles, so the motion can be an arbitrarily small fraction of the total number of vertices (note that the maximum valence is $d=n$ ).

Theorem 3.1 There are only finitely many maps $M$ with $D(M)>2$. There are only finitely many orientable maps with $D^{+}(M)>2$.

Proof. Let $M$ be a map with $n$ vertices and let $A=A u t(M)$. Since the stabilizer of an edge has order at most 4 and there are fewer than $n^{2} / 2$ edges, we have $|A|<2 n^{2}$. If every vertex has the same valence $d>2$, then by Lemma 3.2, $m(A)>n / 6$. Thus, if $n>12 \log _{2}\left(2 n^{2}\right)$, then $D(M) \leq 2$ by the Motion Lemma. So if $n$ is sufficiently large, $D(M) \leq 2$. In particular, there are only finitely many vertex-transitive maps with $D(M)>2$.

Suppose that $M$ is not vertex-transitive and $D(M)>2$. We will show that the maximum valence is at most $d=10$. Let $v$ be an any vertex and let $P$ be its orbit under $A$. Let $X$ consist of all the neighbors of $v$ not in $P$. Then $B=\operatorname{Stab}(v)$ takes $X$ to $X$, acting dihedrally. By the Necklace Problem, if $|X| \geq 6$, then there is a subset $Y \subset X$ such that $\operatorname{Stab}_{B}(Y)$ is trivial. Let $Y^{\prime}=Y \cup\{v\}$. Then $\operatorname{Stab}_{A}\left(Y^{\prime}\right) \subset B$, since no element of $Y$ is in the orbit $P$ of $v$. Thus $\operatorname{Stab}_{A}\left(Y^{\prime}\right)$ is trivial.

Thus each vertex in $M$ is adjacent to at most 5 vertices not in its orbit. It remains to show that at most 5 neighbors of $v$ are in its orbit $P$. Clearly, some neighbor $w$ of $v$ is in a different orbit $Q$, since otherwise every vertex in $P$ is adjacent only to vertices in $P$, making $M$ vertex-transitive or disconnected. Suppose that uvw is a bent angle with $u$ in $P$. Since $\operatorname{Stab}(u, v, w)$ is nontrivial and since $w$ is in a different orbit from $u$ and $v$, there must be an automorphism fixing $w$ and interchanging $u$ and $v$. Thus $w$ is also adjacent to $u$. Since $w$ can be adjacent to at most 5 vertices not in $Q$, there can be at most 4 such bent angles $u v w$, so at most 5 neighbors of $v$ are in $P$.

Thus by part (b) of Lemma 3.2, we have $m(A) \geq 2 n / 100$. Since $|A| \leq 4(10 n / 2)$, there are only finitely many possibilities for $M$ by the Motion Lemma.

If $M$ is orientable, $\operatorname{Aut}^{+}(M)$ is a subgroup of $\operatorname{Aut}(M)$, so $D^{+}(M)=2$ whenever $D(M)=2$.

For a very different approach to showing all but finitely many planar maps have $D(M)=2$, see $[9]$.

\section{The classification of maps with $D^{+}(M)>2$}

Our goal in this section is to classify all maps $M$ with $D^{+}(M)>2$. Recall that for $\mathrm{Aut}^{+}(M)$, we have $\operatorname{Fix}(u, v)$ is trivial for every edge $u v$. We will actually do something much stronger: we will classify all graphs $G$ having a subgroup $A \subset A u t(G)$ that does not fix adjacent vertices and has $D(A, V(G))>2$. As a consequence, we will be able to classify the graphs underlying all maps $M$ with $D(M)>2$ and having no automorphism fixing an edge. 
Theorem 4.1 (Classification of graphs with actions fixing no edge) Let $G$ be a connected graph with vertex set $V$. Suppose that $\operatorname{Aut}(G)$ has a subgroup $A$ fixing no edge such that $D(A, V)>2$. Then $G$ is $C_{3}, C_{4}, C_{5}, K_{4}, K_{5}, K_{7}, O_{6}$ or $O_{8}$.

Proof. Throughout the proof, all automorphisms of $G$ will be assumed to be in the specified group $A$. In particular, "stabilizer" means stabilizer under the action of $A$. We observe that $\operatorname{Stab}(u, v)$ is nontrivial for every edge $u v$, since $D(A, V)>2$. Thus, there must be a unique automorphism $\phi_{u v}$ in $A$ interchanging $u$ and $v$ (it is unique since $|\operatorname{Fix}(u, v)|=1$ so $|\operatorname{Stab}(u, v)|=2)$. In particular, the graph $G$ is vertex-transitive under $A$ and all vertices have the same valence $d$. Also, $D(A, V) \leq 3$ since $\operatorname{Fix}(u, v)$ is trivial. The case $d=2$ leads to the graphs $C_{3}, C_{4}, C_{5}$, so we assume that $d>2$.

We first show that $G=K_{d+1}$ or $O_{d+2}$. Let $v$ be any vertex in $G$ and let $L$ be the link of vertex $v$, namely the subgraph of $G$ induced by the neighbors of $v$. Suppose that $u$ and $w$ are nonadjacent vertices in $L$. Since $\operatorname{Stab}(u, v, w)$ is nontrivial and $u$ is the only vertex in $u, v, w$ that is adjacent to the other two vertices, there must be an element $f$ in $\operatorname{Stab}(v)$ interchanging $u$ and $w$. Moreover, the action of $\operatorname{Stab}(v)$ on $L$ has no fixed vertices, since $\operatorname{Fix}(u, v)$ is trivial for all edges $u v$. Thus, $f$ is the only element of $\operatorname{Stab}(v)$ taking $u$ to $w$ or taking $w$ to $u$. In particular, for any other vertex $x$ in $L$, there is no element of $\operatorname{Stab}(v)$ performing a cyclic permutation of $u, w, x$. Hence, any nontrivial element $g$ of $\operatorname{Stab}(u, v, w, x)$ cannot fix $v$. Since $v$ has valence 3 in the subgraph induced by $u, v, w, x$ and since $u$ and $w$ have valence at most $2, g$ must interchange $v$ and $x$, forcing edges $u x$ and $w x$.

We conclude that $u$ and $w$ are joined to all other vertices in $L$. Therefore every vertex in $L$ has valence either $d-1$ or $d-2$ in $L$. In particular, $L$ is connected. If all vertices of $G$ are adjacent to $v$, then $G=K_{d+1}$, since $G$ is vertex-transitive. If not, there is a vertex $x$ not adjacent to $v$ but adjacent to some $u \in L$. Suppose $w \in L$ is also adjacent to $u$ but not adjacent to $x$. Then $u, v, w, x$ have valences $3,2,2,1$, respectively, in the subgraph of $G$ induced by $Y=\{u, v, w, x\}$, which means any element of $\operatorname{Stab}(Y)$ fixes the edge $u x$, a contradiction. We conclude that $x$ is also adjacent to $w$. By the connectivity of $L$, we have that $x$ is adjacent to all vertices of $L$. Since all vertices of $L$ have valence at least $d-2$ within $L$ together with one edge to $v$ and one to $x$, the graph $G$ consists of $L$ together with $u$ and $x$. The only graph with $d+2$ vertices all of valence $d$ is $O_{d+2}$.

We now restrict the possible values for $d$. Before we proceed, we note that the Motion Lemma alone already restricts the possibilities for $d$. When $G=O_{d+2}$, since $\operatorname{Aut}(M)$ can fix no edge, every element of $A$ moves at least $d$ vertices; also $|\operatorname{Stab}(v)|$ must divide $d$. Thus by the Motion Lemma, when $d>2 \log _{2}(d(d+2))$, we have $D^{+}(M)=2$, so $d<17$. Similarly, for $G=K_{d+1}$, the motion is at least $d$, so again we must have $d<17$. Our arguments will not depend on motion, except in one small case, but it is reassuring to know that no matter what, $d$ must be small. This also means that the remainder of this proof could be replaced by a simple computer calculation.

Suppose that $G=O_{d+2}$. Given $v$, we denote by $v^{*}$ the only vertex in $G$ not adjacent to $v$. Given any edge $u v$, supppose that $w \neq u, v, u^{*}, v^{*}$. Then the nontrivial element $\phi_{u v}$ of $\operatorname{Stab}(u, v)$ must stabilize $\left\{w, w^{*}\right\}$, because any nontrivial element in $\operatorname{Stab}\left(u, v, w, w^{*}\right)$ must interchange $u$ and $v$, as they are the only vertices of valence 3 in the graph induced 
by $u, v, w, w^{*}$ and $\operatorname{Fix}(u, v)$ is trivial. Thus for any vertex $w$, we have that $\operatorname{Stab}\left(w, w^{*}\right)$ includes $\phi_{u v}$ for all $u, v$ in $L_{d}=\operatorname{Link}(w)=\operatorname{Link}\left(w^{*}\right)=O_{d}$. Let $A_{d}$ be the subgroup of $A$ generated by $\phi_{u v}$ for all edges $u v$ in $L_{d}$; note that $A_{d}$ acts transitively on $L_{d}$. Then $\left|\operatorname{Stab}\left(w, w^{*}\right)\right| \geq\left|A_{d}\right|$. Therefore, since $\operatorname{Fix}\left(w, w^{*}\right)$ has index at most 2 in $\left.\operatorname{Stab}\left(w, w^{*}\right)\right)$, we have:

$$
|\operatorname{Stab}(w)| \geq\left|\operatorname{Fix}\left(w, w^{*}\right)\right| \geq\left|A_{d}\right| / 2 .
$$

Let $A_{d+2}$ be the subgroup of $A$ generated by $\phi_{u v}$ for all edges in $O_{d+2}$. Since the action of $A_{d+2}$ is transitive on $d+2$ vertices,

$$
\left|A_{d+2}\right| \geq(d+2)|\operatorname{Stab}(w)| \geq\left|A_{d}\right| / 2 \text {. }
$$

Now we repeat the process inside $L_{d}$ by choosing $x, x^{*}$ in $L_{d}$, with link $L_{d-2}=O_{d-2}$ in $L_{d}$ and group $A_{d-2}$ generated by $\phi_{u v}$ for all edges $u v$ in $L_{d-2}$. Continue the process until $d=4$, at which point $L_{4}=O_{4}=C_{4}$. Then $\left|A_{4}\right| \geq 4$, so $\left|A_{6}\right| \geq 6 \cdot(4 / 2)=12$ and $\left|A_{8}\right| \geq 8 \cdot(12 / 2)=48$. Then for $d=8$, we have $\left|\operatorname{Stab}\left(w, w^{*}\right)\right| \geq 48$, which is impossible since for $d=8,\left|\operatorname{Stab}\left(w, w^{*}\right)\right| \leq 2 \cdot|\operatorname{Stab}(w)| \leq 16$. Since $A_{d}$ grows faster than $2 d$, we have a contradiction for all $d \geq 8$. We also note for later use that for $d=6$, since $\left|A_{6}\right| \geq 12$, then $|\operatorname{Stab}(v)| \geq 6$. In any case, we have that $G=O_{6}$ or $O_{8}$.

Suppose instead that $G=K_{d+1}$. This means every element of $A$ fixes at most one vertex of $G$. Every triangle must have a nontrivial stabilizer, either a 3 -fold rotation or an involution, which necessarily is an edge stabilizer. Our plan is to show that $A$ does not have enough elements of order 2 or 3 to stabilize all $(d+1) d(d-1) / 6$ triangles of $G$, except if $d=3,4,6$. First, we consider involutions stabilizing a triangle (note that this requires that $d+1$ be even since an involution can fix at most one vertex). For each edge $u v$, there is exactly one nontrivial element of $\operatorname{Stab}(u, v)$ and it fixes exactly one vertex $w$, so $u v w$ is the only triangle containing edge $u v$ and stabilized by an involution interchanging $u$ and $v$. Thus the number of triangles stabilized by an involution is at most the number of edges, namely $(d+1) d / 2$. If no triangle is stabilized by an element of order 3 , then

$$
\frac{(d+1) d(d-1)}{6} \leq \frac{(d+1) d}{2}
$$

Therefore, $d \leq 4$.

Thus if $d>4$, there must be some triangles stabilized by elements of order 3 . Since $|\operatorname{Stab}(v)|$ must divide $d$ (as it acts without fixed points on the neighbors of $v$ ) and $|A|=$ $|\operatorname{Stab}(v)|(d+1)$, we must have that 3 divides $d$ or $d+1$. Suppose first that 3 divides $d+1$. Then any automorphism of order 3 fixes no vertex, since it cannot fix more than one vertex. Since $\operatorname{Stab}(u) \cap \operatorname{Stab}(v)=\{1\}$, there are $(d+1)(|\operatorname{Stab}(v)|-1)$ nontrivial elements of $A$ that stabilize some vertex. Since $|A|=(d+1)|\operatorname{Stab}(v)|$, that leaves exactly $d$ elements of $A$ that have no fixed vertices. Thus there are at most $d / 2$ possibilities for automorphisms of order 3 , each stabilizing $(d+1) / 3$ triangles, giving $d(d+1) / 6$ such triangles in all. Since we already know at most $d(d+1) / 2$ are stabilized by involutions, we have

$$
\frac{(d+1) d(d-1)}{6} \leq \frac{(d+1) d}{2}+\frac{(d+1) d}{6} .
$$


Therefore, $d \leq 5$ and hence $d=5$, since 3 divides $d+1$.

Finally suppose that 3 divides $d$. Then each element of order 3 fixes exactly one vertex. Assume for the moment that $\operatorname{Stab}(v)$ is cyclic, which would be the case if $A$ were $\operatorname{Aut}^{+}(M)$ for a map $M$. Then there is at most one element of order 3 (other than its inverse) in $\operatorname{Stab}(v)$ and it stabilizes $d / 3$ triangles, so there are at most $(d+1) d / 3$ triangles in all that are stabilized by elements of order 3 . It follows that

$$
\frac{(d+1) d(d-1)}{6} \leq \frac{(d+1) d}{3}+\frac{(d+1) d}{2} .
$$

Therefore, $d \leq 6$ and hence $d=3$ or $d=6$.

For the remaining cases, where $d=5$, or where 3 divides $d$ and $\operatorname{Stab}(v)$ is not cyclic, we need a little group theory. The action of $A$ on the $d+1$ vertices makes $A$ a Frobenius group, that is a transitive permutation group with no nonidentity element fixing more than one symbol. Frobenius groups have highly restricted structure (see [8]), but we only need that $A$ is a semi-direct product of the Frobenius kernel $F$ of order $d+1$ and $\operatorname{Stab}(v)$, such that the natural homomorphism of $\operatorname{Stab}(v)$ into $\operatorname{Aut}(F)$, given by conjugation, is an injection. Note that $|\operatorname{Stab}(v)|$ divides $d$ since it acts on $d$ points leaving none fixed. By the Motion Lemma, we have $d<17$, so the only cases we need consider are $d=9,12,15,5$. The case $d=9$ cannot occur since $\operatorname{Aut}(F)$ has no elements of order 3, for the two possibilities $F=Z_{10}$ or $F=D_{5}$. For $d=12, F$ is cyclic of order 13 making $\operatorname{Stab}(v)$ cyclic, and for $d=15, \operatorname{Stab}(v)$ is cyclic since its order divides 15 . Finally, we note that the case $d=5$ cannot occur since $\operatorname{Aut}(F)$ has no elements of order 5 , for the two possibilities $F=Z_{6}$ or $F=D_{3}$.

We conclude that $d=3,4,6$ for the case that $G=K_{d+1}$.

Corollary 4.1 Any Frobenius group on $n$ symbols, for $n \neq 4,5,7$, has distinguishing number 2 .

We now give the full classification of maps with $D^{+}(M)=3$. The classification for maps with $D(M)=3$ is far more complicated and is given in a sequel to this paper. We need to be able to describe algebraically some fairly complicated maps and the easiest way is with the terminology and notation of Cayley maps [17]. Given a group $A$ and generating set $W$, the Cayley graph $C(A, W)$ is the directed, labeled graph with a vertex set $A$ and directed edge labeled $w$ from $a$ to $a w$ for each $a$ in $A$ and $w$ in $W$ (if $w$ is an involution, the edges from $a$ to $a w$ and from $a w$ to $a$ are identified to a single undirected edge). We also refer to the undirected, unlabeled graph as a Cayley graph. Note that each edge incident to a given vertex $a$ can be labeled with an element of $w \in W$ or its inverse, depending on whether the corresponding directed edge labeled $w$ begins or ends at $a$.

Given a Cayley graph $C(A, W)$ and a cyclic ordering $\rho$ of the elements in $W \cup W^{-1}$, the Cayley map $C M(A, \rho)$ is the orientable map whose underlying graph is $C(A, W)$ and the rotation of labeled edges at each vertex is given by $\rho$. We denote $\rho$ simply by a cycle in parenthesis. Left multiplication by an element of $A$ is clearly a graph automorphism, but it 
is also a map automorphism since it preserves the cyclic order of edges incident to a vertex. Thus, the map is vertex-transitive. In addition, any group automorphism that permutes the elements of $W \cup W^{-1}$ and preserves the cyclic order $\rho$ induces an orientation-preserving automorphism of the Cayley map. In particular, suppose that $A$ is the cyclic group $Z_{n}$ written additively, $r$ is a root of $-1(\bmod n)$, and $\rho=\left(1, r, r^{2}, \cdots,-1,-r,-r^{2} \cdots\right)$. Then multiplication by $r^{i}$ is a map automorphism of $C M\left(Z_{n}, \rho\right)$ for all $i$.

Theorem 4.2 If $D^{+}(M)=3$ and $M$ has no vertex of valence 1 or 2 , then $M$ is the triangular embedding of $K_{4}$ in the sphere (the tetrahedron as a map), the triangular embedding of $\mathrm{O}_{6}$ in the sphere (the octahedron as a map), $C M\left(Z_{5},(1,2,-1,-2)\right.$ ) or $C M\left(Z_{7},(1,3,2,-1,-3,-2)\right)$. The last two are the quadrangulation and triangulation of the torus by $K_{5}$ and $K_{7}$, respectively.

Proof. We first show that the four maps have $D^{+}(M)=3$. For the tetrahedron, this is easily verified. For the octahedron, we must check that every set of two or three vertices has a nontrivial stabilizer. This is easily verified for two vertices. Any three vertices either form a triangle or a path uvw of length 2 joining nonadjacent vertices; the first is stabilized by a 3-fold rotation and the second by a half-turn about $v$. For $M=C M\left(Z_{5},(1,2,-1,-2)\right)$, we need only check that sets of at most two vertices are stabilized. Multiplication by 2 preserves the rotation and hence is a map automorphism performing a 4-fold rotation about vertex 0. Thus, Stab(0) is nontrivial and $A u t^{+}(M)$ acts transitively on edges, so we need only find a stabilizer for $\{0,1\}$ : multiplication by -1 followed by addition of 1 works. For $M=C M\left(Z_{7},(1,3,2,-1,-3,-2)\right)$, we must check sets of size at most 3. Again, multiplication by 3 preserves the rotation and hence is a map automorphism, giving a 6 -fold rotation about 0 . Thus for sets of size 2 , we need only check $\{0,1\}$ : multiplication by -1 and addition of 1 works. For sets of size 3 , by the 6 -fold rotation, we need only check the sets $\{0,1,3\},\{0,1,2\}$ and $\{0,1,-1\}$. The first is stabilized by multiplication by 2 followed by addition of 1 , the second by multiplication by -1 followed by addition of 2 , and the third by multiplication by -1 .

Next we show the given maps are the only possibilities. The action of $\operatorname{Aut}^{+}(M)$ fixes no edges, so the underlying graph of $M$ is $K_{4}, K_{5}, K_{7}, O_{6}$ or $O_{8}$. We first eliminate $O_{8}$. There are 32 triangles to be stabilized. Suppose that the triangle $u v w$ is stabilized by an involution in $\operatorname{Stab}(v)$. In $\operatorname{Aut}^{+}(M)$, the only possibility is that $u v w$ is straight since there are no reflections for bent angles. On the other hand, all 12 bent angles at $v$ are closed (again since there are no reflections for bent angles) and there are only 12 edges in $\operatorname{Link}(v)$. Thus all straight angles at $v$ are open, a contradiction of $u v w$ being a triangle. We conclude that all 32 triangles are stabilized by elements of order 3. Any element of order three must fix exactly two vertices, which must be nonadjacent, say $v$ and $v^{*}$ (since the $A u t^{+}(M)$ fixes no edges). Since $\operatorname{Stab}(v)=\operatorname{Stab}\left(v^{*}\right)$ has at most one elment of order 3 and it stabilizes two triangles, we can stabilize only $4 \cdot 2=8$ triangles this way.

It remains to show the maps with underlying graphs $K_{4}, O_{6}$ and $K_{7}$ are triangulations and the map with underlying graph $K_{5}$ is a quadrangulation (see [12] for the uniqueness of these embeddings). Note at the outset that the presence of the half-turn $\phi_{u v}$ for each edge $u v$ guarantees that the map is face-transitive, so all faces have the same size. We 
also note that every bent angle, and hence every corner, is closed, since the stabilizer in $\mathrm{Aut}^{+}(M)$ of an open bent angle is trivial. In particular, if uvw is a corner, then uvw is a triangle in the underlying graph. If both $v w u$ and $w u v$ are bent angles, that triangle is a face, since any stabilizer $f$ of $\{u, v, w\}$ cannot fix $u, v$, or $w$, so $f$ is 3 -fold rotation, making $v w u$ and $w u v$ also corners.

For $K_{4}, O_{6}$ and $K_{7}$, we need a triangular face. There are no straight angles in a map for $K_{4}$, so by the preceding remarks about corners, the faces are triangles. For $O_{6}$, there are $4 \cdot 6=24$ map corners, each in a triangle, and 8 triangles; thus all corners of triangles are face corners, so every face is a triangle.

For $K_{7}$, label all angles $b, c$ or $s$, depending on whether the angle is bent but not a corner, a corner, or straight. If there is no triangle labeled $c c c$, then any triangle with one $c$ must be $c c s$ in order to be stabilized by an involution. There are $7 \cdot 6=42$ angles labeled $c$, so there must be 21 triangles labeled ccs. The other 14 triangles must all be labeled $b b b$, since there are also 42 angles labeled $b$. Suppose $u x v$ and $v x w$ are adjacent corners, and consider all the angles in the $K_{4}$ subgraph $H$ determined by $u, v, w, x$. Then triangles $u x v$ and $v x w$ are both ccs. Thus of the 12 angles in $H$, at least 4 are $c$ and 2 are $s$. On the other hand, at any vertex of $H$, at least one of the three angles in $H$ around the vertex is $b$ (since the valence is 6 so they cannot be $c c c, s s s, c s s$, or $c c s$ ). Thus at least 4 angles of $H$ are labeled $b$. Since all triangles with an angle labeled $b$ are $b b b$, the number of $b$ angles must be divisible by 3 . Hence, there must be at least 6 angles labeled $b$. Therefore, at some vertex of $H$, two of the three angles are labeled $b$, which means the third angle is also $b$, since the valence is 6 . Each of these angles is in a different triangle of $H$, so there must be 9 angles in $H$ labeled $b$, a contradiction since we already have 4 angles labeled $c$. We conclude that at least one triangle is labeled $c c c$, so all faces are triangles.

For $K_{5}$, let $m$ be the number of faces. Since all faces have the same size, $m$ divides 20 (twice the number of edges) and $m$ is odd (since the Euler characteristic of an orientable surface is even). The only possibilities are $m=1$ and $m=5$. If $m=1$, then $A u t^{+}(M)$ is a subgroup of the cyclic group $Z_{20}$, since all automorphisms must rotate the single face around its center. But then there is only one involution which stabilizes at most 2 edges. We conclude that $m=5$, so the face sizes are all 4 .

The following example shows that the final possibility of Theorem 4.1 is realized by a map automorphism group, but only by including orientation-reversing automorphisms. A Cayley map $C M(A, \rho)$ is called balanced if all generators are involutions or $w$ and $w^{-1}$ are antipodal in the cyclic order $\rho$ for all $w \in W$ (see [17]). An important fact about balanced Cayley maps is that every map automorphism fixing the identity is also a group automorphism $([23])$

Example 4.1 Let $M$ be the Cayley map $C M(Q,(i, j, k,-i,-j,-k))$, where $Q$ is the quaternions. The underlying Cayley graph is $\mathrm{O}_{8}$, with multiplication by -1 providing the antipodal automorphism. The group automorphism $f$ interchanging $i$ with $j,-i$ with $-j$, and $k$ with $-k$, is an orientation-reversing map automorphism since it reverses the rotation $(i, j, k,-i,-j,-k)$. A similar map automorphism $g$ interchanges $j$ with $k$, so 
Stab(1) contains the dihedral group $D_{3}$. On the other hand, there is no group automorphim fixing $i$, so there is no map automorphism fixing the edge $1 i$ (since the Cayley map is balanced). Thus the action of $\operatorname{Aut}(M)$ fixes no edges and $\operatorname{Stab}(1)=D_{3}$. We claim $D(M)=3$. We must show that $\operatorname{Stab}(Y)$ is nontrivial for every vertex subset $Y$; we can assume $|Y| \leq 4$ using complements. For $|Y|=2$, the automorphism $f$ shows how to interchange adjacent vertices, and multiplication by -1 interchanges antipodal vertices. For $|Y|=3$, if $Y$ contains antipodal points, it behaves like $1, k,-k$ which is stabilized by $f$, and if all vertices of $Y$ are mutually adjacent, it behaves like $1, i, j$, which is stabilized by $f$. For $|Y|=4$, if $Y$ contains two pairs of antipodal points, it is stabilized by multiplication by -1 . If $Y$ contains one pair of antipodal points, it behaves like $1,-1, i, j$, which is stabilized by $f$. If $Y$ contains no antipodal points, it behaves like $1, i, k,-j$, which is stabilized by $g f$.

\section{Chromatically distinguishing maps}

For automorphisms of graphs, it is natural to ask how the distinguishing number is affected if colorings are required to be proper, that is adjacent vertices get different colors. Collins and Trenk [6] call this the distinguishing chromatic number of a graph $G$, denoted $\chi_{D}(G)$, that is the smallest number $k$ such that $G$ can be properly colored with $k$ colors so that no automorphism of $G$ preserves the coloring. In the same way, we define the distinguishing chromatic number of a map $M$, denoted $\chi_{D}(M)$, only now the automorphisms are map automorphisms; the orientation-preserving distinguishing number, denoted $\chi_{D^{+}}(M)$, is defined similarly for an orientable map $M$. We also denote by $\chi(G)$ the usual chromatic number of a graph $G$ and by $\chi(M)$ the chromatic number of the graph underlying the $\operatorname{map} M$.

By definition, $\chi_{D} \geq \chi$, for both graphs and maps. For graphs, $\chi_{D}(G)$ can be much bigger than $\chi(G)$. For example, for the complete bipartite graph $K_{m, n}$, we clearly have $\chi\left(K_{m, n}\right)=2$ and $\chi_{D}\left(K_{m, n}\right)=m+n$. On the other hand, for maps we have:

Proposition 5.1 For any map $M$, we have $\chi_{D}(M) \leq \chi(M)+3$. When $M$ is orientable, $\chi_{D^{+}}(M) \leq \chi(M)+2$.

Proof. Suppose that $M$ has been properly colored with $k=\chi(M)$ colors. Choose any corner $u v w$ in the map and change the colors of vertices $u, v$, and $w$ to three new extra colors. Then the map has no color-preserving automorphism. In the orientable case, if we only wanted to destroy orientation-preserving automorphisms, we would only have to add two colors, one for $u$ and one for $v$, since that would fix the edge $u v$.

Example 5.1 For $n>3$, let $R_{n}$ be the double pyramid (or anti-prism) as a map in the sphere with a cycle $C_{n}$ at the equator, all vertices joined to vertices at the north and south pole. Then $\chi\left(R_{n}\right)=3$ for $n$ even and $\chi\left(R_{n}\right)=4$ for $n$ odd. It is easy to check that $\chi_{D}\left(C_{4}\right)=\chi_{D}\left(C_{6}\right)=4$ and $\chi_{D}\left(C_{n}\right)=3$ for all other $n$. Since the north and south poles must get different colors from each other or else reflection in the equator is color-preserving, we have: 
- $\chi_{D}\left(R_{n}\right)=\chi\left(R_{n}\right)+3=6$ for $n=4,6$;

- $\chi_{D}\left(R_{n}\right)=\chi\left(R_{n}\right)+2=5$ for all even $n>6$;

- $\chi_{D}\left(R_{n}\right)=\chi\left(R_{n}\right)+1=5$ for all odd $n$.

Thus there are maps with $\chi_{D}(M)=\chi(M)+3$. Such maps, however, are few and highly restricted.

Theorem 5.1 Suppose that $\chi_{D}(M)=\chi(M)+3=k+3$. Then $k$ must be 2 or 3 . Moreover, there are only finitely many such maps $M$.

Proof. Color $M$ properly with $k$ colors. Let $u v$ be any edge. Recolor $u$ and $v$ with two new extra colors. Since $\chi_{D}(M) \neq \chi(M)+2$, there must be a color-preserving reflection across the edge $u v$. Since the edge $u v$ is arbitrary, the original coloring of the map is colorpreserving face-transitive. Notice this implies that the vertices in the rotation about any vertex are either all the same color or alternate between two colors.

Color $M$ with $k$ colors so that color 1 is used the least among all proper colorings of $M$ with $k$ colors. Suppose that $k>3$. Since the vertices adjacent to any vertex are all the same color, or alternate between two colors, any vertex labeled 1 can be recolored a different color, reducing the number of vertices colored 1 and contradicting the minimal property of the coloring and the color 1 . Thus $k$ is 2 or 3 .

Suppose $k=2$. We claim that the maximum valence $d \leq 5$. Indeed, suppose $v$ has valence $d>5$. Then by the Necklace Problem, we could recolor $v$ with a new color 3 and some neighbors with a new color 4 so that the new coloring has no color-preserving automorphisms. Since $d \leq 5$, by Lemma 3.2 and the Motion Lemma, $D(M)=2$ for all but finitely many $M$. If $D(M)=2$, suppose that $\operatorname{Stab}(Y)$ is trivial. Let $Y_{i}$ be the subset of $Y$ colored $i$, for $i=1,2$. Coloring $Y_{1}$ with new color 3 and $Y_{2}$ with new color 4 , we conclude that $\chi_{D}(M)=4$. Thus only finitely many $M$ with $k=2$ have $\chi_{D}(M)=k+3=5$.

Suppose instead that $k=3$. Given a proper three-coloring of $M$ such that 1 is the least used color over all proper three-colorings, let $M^{\prime}$ be the map induced by vertices colored 2 or 3 . We first claim that $M^{\prime}$ is connected. Since 1 is least used over all proper three-colorings, each vertex colored 1 must have neighbors with alternating colors, else it could be recolored 2 or 3 . Let $u v w$ be an open bent angle with $u, v, w$ colored $2,1,3$, respectively. If we recolor $v$ with 4 and $u$ and $w$ with 5 , since $\chi_{D}(M) \neq 5$, there is a color-preserving automorphism interchanging $u$ and $w$, but this is impossible since the remaining vertices in the rotation at $v$ alternate 2 and 3 . We conclude each bent angle around $v$ is closed, so $\operatorname{Link}(v)$ is contains a cycle through all neighbors of $v$ when $v$ is a branch vertex. If $v$ is colored 1 and has valence 2, there must be a path in $M^{\prime}$ connecting the two neighbors of $v$ or else one could switch colors 2 and 3 in one component of $M^{\prime}$ so that both neighbors of $v$ have color 2. Then $v$ could be recolored 3, contradicting the minimality of the color 1 . It follows that any path in $M$ between vertices in $M^{\prime}$ can be modified to avoid vertices of color 1 , so $M^{\prime}$ is connected.

By the case $k=2$, we know if $M^{\prime}$ has enough vertices, it has a proper four-coloring with no color-preserving automophisms. When we put back in the vertices of the original 
map $M$ colored 1, we get a proper five-coloring of $M$ such that that any color-preserving automorphism fixes all vertices in $M^{\prime}$. If some vertex of $M^{\prime}$ is a branch vertex (in $M^{\prime}$ ), such an automorphism must fix a bent angle and hence be the identity.

Therefore we assume that the graph underlying $M^{\prime}$ is a cycle or path of length $n$ properly colored with 2 and 3. Then for $n>6$, we can add one color to the coloring of $M^{\prime}$, so that the any color-preserving automorphism is a reflection $f$ fixing the vertices of $M^{\prime}$. If $v$ is colored 1 and not fixed by $f$, color $f(v)$ with a second new color which makes $f$ not color-preserving. If instead $f$ also fixes all vertices colored 1 , then the original map is a cycle, so $\chi_{D}(M) \neq \chi(M)+3$.

We have already seen that $\chi_{D}(M)=\chi(M)+3$ for $M=R_{4}$ and $M=R_{6}$. Even if these are not the only maps with $\chi_{D}(M)=\chi(M)+3$, it should be possible to classify the finite number of such maps, using ideas in the proof of Theorem 5.1. For example, if $k=2$, we would require $D(M)=3$ and by the sequel to this paper the only possibilities are $K_{m, n}$ for $m, n=3,4,5$. If $k=3$, we would only need to consider graphs obtained by adding 5 or fewer vertices to such $K_{m, n}$. Even though $\chi_{D}(M)=\chi(M)+2$ for infinitely many maps (e.g $R_{n}$ for all even $n>6$ ), we suspect such maps can also be classified.

Collins and Trenk have conjectured that $\chi_{D}(G) \leq 6$ for all 3-connected planar graphs:

Corollary 5.1 Let $M$ be a planar map. Then $\chi_{D}(M) \leq 6$. Thus, $\chi_{D}(G) \leq 6$ for any 3-connected planar graph $G$. Moreover, if $\chi(M)=3$ and $\chi_{D}(M)=6$, the only possibilities are $R_{4}$ and $R_{6}$.

Proof. The first statement follows from the Four Color Theorem and Theorem 5.1. The second follows from the uniqueness of 3-connected planar embeddings [12], which implies that for any planar embedding of $G$, every graph automorphism is also a map automorphism. For the final statement, let $M^{\prime}$ be as in the proof of Theorem 5.1. If $M^{\prime}$ has a branch vertex and if $D\left(M^{\prime}\right)=2$, then as before $M$ has a proper five-coloring with no color preserving autormophism. If $M^{\prime}$ has no branch vertex, then as before, we have $\chi\left(D(M) \neq \chi(M)+3\right.$, unless the map is $R_{4}$ or $R_{6}$. Suppose instead that $M^{\prime}$ has a branch vertex and $D\left(M^{\prime}\right)=3$. By the sequel to this paper, the only such planar bipartite map with $D\left(M^{\prime}\right)=3$ is the cube. The color 1 is then located at the centers of the faces of the cube. Since the action of $\operatorname{Aut}\left(M^{\prime}\right)$ on the faces is faithful and equivalent to the action of $\operatorname{Aut}\left(O_{6}\right)$ on its vertices, we can replace color 1 by three new colors to get a five-coloring with no color-preserving automorphism.

Sakurai has also shown that $\chi_{D}(G) \leq 6$ for 3-connected planar graphs [20].

Recall that $\chi_{D}\left(R_{n}\right)=\chi\left(R_{n}\right)+2$ for all even $n>6$. Are there other planar maps with $\chi_{D}(M)=\chi(M)+2$ ? For example, if $n$ is even and alternate spokes to the north and south pole are deleted, the resulting map has $\chi=2$ and $\chi_{D}=4$. One can also get examples by deleting equator edges of $R_{n}$ (an extra color is needed along the equator to eliminate a reflection fixing the poles). With finitely many exceptions, these are the only examples:

Theorem 5.2 There are only finitely many planar maps $M$ such that $\chi_{D}(M)=\chi(M)+2$ and such that $M$ is not obtained from $R_{n}$ for some $n$ by deleting some edges. 
Proof. Let $M$ be a planar map with $\chi_{D}(M)=\chi(M)+2=k+2$. Given a proper coloring of $M$ with $k$ colors, let $A$ be the subgroup of $\operatorname{Aut}(M)$ that preserves the coloring. Suppose that 1 is the most frequently used color and let $X$ be the vertices colored 1 . By [7], we have that $D(A, X)=2$ whenever $|X|>6$. Thus if the action of $A$ on $X$ is faithful, we can find a subset $Y \subset X$ such that the stabilizer of $Y$ in $A$ is trivial. Color the vertices of $Y$ be a new color and we have $\chi_{D}(M)=\chi(M)+1$.

Suppose instead the action of $A$ is not faithful on $X$ and that $f \in A$ fixes all vertices in $X$. The finite groups acting on the sphere are well-known [12] and the only nonidentity automorphism fixing more than two points is a reflection. Thus, $f$ is reflection in a circle $C$ containing $X$. Let $v$ be a vertex not on $C$ and let $Y$ be the vertices in $X$ not adjacent to $v$. If $|Y|>5$, then by the Necklace Problem we can introduce an extra color for some of the vertices in $Y$ so that the only color-preserving element of $A$ must be the reflection $f$ fixing $C$ and which therefore must move $v$. Since $v$ is not adjacent to any vertex in $Y$, we can change its color also to the extra color and eliminate $f$ as a color-preserving automorphism. Thus $\chi_{D}(M)=\chi(M)+1$. If $|X|>12$, it follows that any two vertices in the same component of $S-C$ are adjacent to at least three common vertices in $X$, forming a copy of $K_{2,3}$ in one component of $S-C$. Using the reflection $f$, we get a copy of $K_{4,3}$ embedded in the sphere, a contradiction.

We conclude that there is only one vertex $v$ in each component of $S-C$, as long as $|X|$ is sufficiently large. Suppose there is an edge between $v$ and $f(v)$. Then $v$ and $f(v)$ already have different colors, so $\chi_{D}(M)=\chi(M)+1$, since we only need to add one extra color to distinguish any automorphism fixing $X$, for sufficiently large $|X|$. If there are no edges between $v$ and $f(v)$, we have $R_{n}$ with some edges deleted.

Corollary 5.2 There are only finitely many planar maps with $\chi_{D}(M)=6$.

Proof. Theorems 5.1 and 5.2, together with the Four Color Theorem, imply that we need only consider maps obtained by deleting edges from $R_{n}$. As we have already observed, $\chi_{D}\left(C_{n}\right)=3$ for $n \neq 4,6$, so we can always use 3 colors along the equator to distinguish any automorphism other than the reflection interchanging the poles. Thus $\chi_{D}(M) \leq 5$ for all such maps, if $n \neq 4,6$.

We conjecture that, in fact, the only planar maps with $\chi_{D}(M)=6$ are $R_{4}$ and $R_{6}$. By Corollary 5.1, we need only consider maps with $\chi(M)=4$. S. Sakurai has shown this is true if the planar map is a triangulation [20].

We observe that the argument of Theorem 5.2 can be applied to maps in higher genus surfaces:

Theorem 5.3 There are only finitely many 3-connected maps in the surface of genus $g$ such that $\chi_{D}(M)=\chi(M)+2$ and such that the underlying graph for $M$ is not $R_{n}$ for $n$ even, or $R_{n}-e$ with $n$ odd and $e$ an edge on the equator.

Proof. We sketch a proof. Let $A$ and $X$ be as in the proof of Theorem 5.2. Note that $\chi(M) \leq H(g)$, the Heawood number for $S$ [12], so if $|X|$ is bounded above, so is the 
number of vertices of $M$. For the part where $D(A, X)=2$, we need $|X|>n(g)$ where $n(g)$ is a number depending on the genus $g$ [7]. We need 3-connectedness, since a reflection can have more than one fixed circle when $g>0$; this also eliminates all the subgraphs of $R_{n}$ except $R_{n}-e$. For the non-faithful case of the proof, it can be shown that if $|X|$ is sufficiently large (again depending on $g$ ), then the only automorphism fixing $X$ is a reflection (see [7]).

We note that for $g>1$, this result might be expected since the Riemann-Hurwitz equation [12] gives $|A| \leq 168(g-1)$, so there are only finitely many possible automorphism groups. Note that since $n(g)$ increases with $g$, as well as the number of points needed to guarantee a reflection, we do not have that there are only finitely many maps $M$ with $\chi_{D}(M)=\chi(M)+2$.

The same ideas give a similar result for $\chi_{D^{+}}$:

Theorem 5.4 There are only finitely many maps $M$ in the surface of genus $g$ such that $\chi_{D^{+}}(M)=\chi(M)+2$.

Proof. Again, let $A$ and $X$ be as in the proof of Theorem 5.2, except now all automorphisms in $A$ preserve orientation. As before, if $|X|$ is sufficiently large, then $D(A, X)=2$, but in this case there is no reflection fixing the vertices of $X$. Thus the action of $A$ on $X$ is faithful, so we simply introduce an extra color for the vertices of $X$ so that no element of $A$ preservers the coloring.

\section{$6 \quad$ Distinguishing graphs and other problems}

Distinguishing graphs, as opposed to maps, has much more flexibility, since vertex and edge stabilizers have few restrictions under graph automorphisms. Nevertheless, the situation for maps suggests some approaches for graphs. Since distinguishability is sensitive to local structure (e.g attaching a complete graph at a vertex), it is best to concentrate on vertex-transitive graphs. An agenda for graphs would be the following general problem:

Problem. Find interesting classes of vertex-transitive graphs such that all but finitely many graphs $G$ in the class have $D(G) \leq 2$.

The following example illustrates some of the issues.

Example 6.1 The wreath graph $W_{n}$ is the lexicographic product of $C_{n}$ with the interval $K_{2}$; that is, $W_{n}$ has two $n$-cycles $u_{1}, \cdots, u_{n}$ and $v_{1}, \cdots v_{n}$ with additional edges from $u_{i}$ to $v_{i-1}$ and to $v_{i+1}$ for all $i$, where subscripts are treated modulo $n$. Then for every $i$ there is an automorphism $f_{i}$ of $W_{n}$ that interchanges the vertices $u_{i}$ and $v_{i}$ and leaves all other vertices fixed. In particular the motion of the automorphism of group of $W_{n}$ is 2 for all $n$. Although $W_{n}$ could still be 2-distinguishable for $n>5$, it is not. Indeed, any 2-coloring of $W_{n}$ that is not 2-distinguishable must assign different colors to $u_{i}$ and $v_{i}$, for each $i$. Rotate the graph one notch (adding one modulo $n$ to the subscript of each vertex); then whenever $u_{i+1}$ is colored differently from $u_{i}$, apply the automorphism $f_{i}$. Although 
each of these automorphisms may not preserve colors, their composition is a nontrivial color-preserving automorphism.

We observe that all the graphs of this example embed in the torus, but almost none of the automorphisms of the graph extend to the torus. This shows how any application of map-distinguishability to graph-distinguishability is likely to be restricted.

One way to use our map results for graphs is to impose conditions such that the graph is the underlying graph of a map whose automorphism group is the same as that of the graph. Negami calls such a graph embedding faithful [15]. This happens for 3-connected planar graphs, or for 3-connected graphs having large face-width [18] or large edge-width embeddings [14].

Another idea motivated by maps is to impose restrictions on set stabilizers, especially vertex or edge stabilizers, to obtain all-but-finitely-many results. For example, [22] characterizes graphs underlying vertex-transitive maps solely in terms of stabilizers of vertices and edges. We observe that Theorem 3.1 classifies graphs, not maps, with actions fixing no edge and having distinguishing number 3. We also note that Theorem 3.1 can be applied to any group $A$ acting faithfully on a set $X$. For example, given $(A, X)$, let $G$ be the graph with vertex set $X$ and with edges $x y$ whenever $\operatorname{Fix}(x, y)$ is trivial. Then by Theorem 3.1, if $G$ is connected and spans $X$, then $D(A, X)=2$, if $|X|>8$.

Finally, we observe that simply limiting the size of the group $A$, compared to the size of the set $X$ it acts upon, can be enough, when the action is transitive. In [7], it is shown that if $(A, X)$ is transitive and $|A|<2^{\sqrt{n}}$, where $n=|X|$, then $D(A, X)=2$. Since $|\operatorname{Aut}(M)| \leq 2 n^{2}$ for any map $M$, where $n=|V|$, this can be viewed as a generalization of our result that all but finitely many transitive maps have $D(M) \leq 2$.

We conclude with some problems for future study.

A basis for a faithful action $(A, X)$ is a subset $B \subset X$ such that Fix $(B)$ is trivial [3]. Thus, if two elements of $A$ agree on the set $B$, they agree everywhere. A Frobenius action is a transitive action with a 2-basis and every edge of an orientable map $M$ is a basis for $\mathrm{Aut}^{+}(M)$ acting on $V(M)$. Since no map automorphism fixes a bent angle, maps with a branch vertex always have a 3-basis.

Problem: Classify faithful transitive actions $(A, X)$ having a 3 -basis and $D(A, X)>2$.

The number of such transitive actions is known to be finite [7].

Problem: Classify transitive faithful actions $(A, X)$ having cyclic point stabilizers and $D(A, X)>2$.

Theorem 3.1 does this in the case where the action is a Frobenius group. From the Motion Lemma [7], we also know that $|X| \leq 43$.

Problem: Study the distinguishability of other combinatorial geometric structures, such as polytopes or triangulated $n$-manifolds.

There are a number of problems coming from distinguishing chromatic number for maps.

Problem: Classify all maps $M$ with $\chi_{D}(M)=\chi(M)+3$.

Problem: Classify all planar maps $M$ with $\chi_{D}(M)=\chi(M)+2$.

Problem: For small genus $g$, classify all maps $M$ with $\chi_{D}(M)=\chi(M)+2$. 
Problem: Prove that the only planar maps $M$ with $\chi_{D}(M)=6$ are the double pyramids $R_{4}$ and $R_{6}$.

Problem: Classify the maps $M$ such that $\chi_{D^{+}}(M)=\chi(M)+2$.

Finally, we close with a conjecture about infinite graphs, which we have asked in various talks, but never in print.

Conjecture: Let $G$ be a locally finite, infinite graph. If $A=\operatorname{Aut}(G)$ acting on $V(G)$ has infinite motion, then $D(A, V(G))=2$.

The conjecture is true for trees [26]. It is also true, without the requirement of infinite motion, when $A$ is countably infinite (unpublished). Note that this means $D(M)=2$ for any locally finite map, since $A u t(M)$ is countable (there are countably many edges and each edge has finite stabilizer).

\section{References}

[1] M. Albertson, Distinguishing cartesian powers of graphs, Electron. J. Combin. 12 (2005), N17.

[2] M. Albertson and K. Collins, Symmetry breaking in graphs, Electron. J Combin. 3 (1996), R18.

[3] R. Bailey and P. Cameron, Base size, metric dimension and other invariants of groups and graphs, preprint.

[4] M. Chan, The maximum distinguishing number of a group, Electron. J. Combin.13(1) (2006), R70.

[5] M. Chan, The distinguishing number of the direct product and wreath product actions, J. Algebraic Comb.24 (2006), 331-345.

[6] K. Collins and A. Trenk, The distinguishing chromatic number, Electr. J. Comb 13(1) (2006), R16

[7] M. Conder and T. Tucker, Motion and distinguishing number two, preprint.

[8] J. Dixon and B. Mortimer, Permutation Groups, Springer, 1996.

[9] T. Fukuda, S Negami, T. Tucker, 3-Connected planar graphs are 2-distinguishable with few exceptions, Yokohama Math J., 54 (2008), no. 2, 143-153.

[10] D. Gluck, Trivial set-stabilizers in finite permutation groups, Canad. J. Math 35 (1983), 59-67.

[11] C. Godsil, Distinguishing primitive permutation groups, arXiv: 0806.2078v1 (2008).

[12] J. Gross and T. Tucker, Topological Graph Theory, Wiley 1987 and Dover 2000.

[13] M. Imrich and S Klavžar, Distinguishing cartesian powers of graphs, J. Graph Theory 53 (2006), 250-260.

[14] B. Mohar and C. Thomassen, Graphs on Surfaces, Johns Hopkins University Press, 2001. 
[15] Seiya Negami, Distinguishing numbers and faithfulness of embeddings of graphs in surfaes, Abstract for 20th Workshop in Topological Graph Theory in Yokohama, 2008.

[16] Seiya Negami, Distinguishing numbers and re-embedding structures of triangulations on closed surfaces, Electronic Notes Disc. Math. 31 (2008), 227-230.

[17] R.B. Richter; J. Širàn̆, R. Jajcay, T. Tucker, M. Watkins, Cayley maps, J. Combin. Theory Ser. B 95 (2005), 189-245.

[18] N. Robertson and R. Vitray, Representativity of surface embeddings, Paths, Flows and VLSI-layout (Bonn 1988), 293-328, Algoriths Combin 9, Springer, Berlin, 1990.

[19] A. Russell and R. Sundaram, A note on the asymptotics and computational complexity of graph distinguishability, Electron. J. Combin. 5(1) (1998), R23.

[20] S Sakurai, Distinguishing chromatic numbers of planar graphs, Abstract for 20th Workshop on Topological Graph Theory in Yokohama, 2008.

[21] A. Seress, Primitive permutation groups with no regular orbits on the set of subsets, Bull. London Math. Soc. 29 (1997), 697-709.

[22] J. Širáň and T. Tucker, Characterization of graphs which admit vertex-transitive embeddings, J. Graph Theory 55 (2007), 233-248.

[23] J. Širáň and T. Tucker, Symmetric maps, in Topics in Topological Graph Theory, R. Wilson and L. Beineke, eds, Cambridge University Press, 2009.

[24] W. T. Tutte, What is a map? in: New Directions in the Theory of Graphs, Acad. Press, N.Y., 1973, 309-325.

[25] J. Tymoczko, Distinguishing numbers for graphs and groups, Electron. J. Combin. 11(1) (2004), R63.

[26] M. Watkins, X. Zhou, Distinguishability of locally finite trees, Electron. J. Combin. 14 (2007), R29.

[27] T-L Wong and X. Zhu, Distinguishing labeling of group actions, Disc. Math. 309 (2009), 1760-1765. 